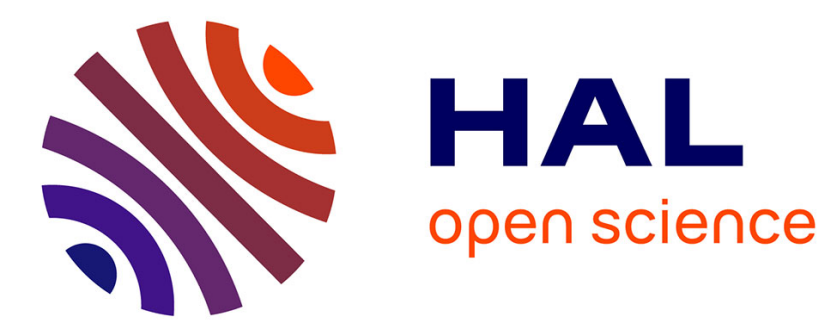

\title{
LIGHT SCATTERING AND REFLECTIVITY OF LIQUID INTERFACES
}

\author{
D. Langevin, J. Meunier
}

\section{To cite this version:}

D. Langevin, J. Meunier. LIGHT SCATTERING AND REFLECTIVITY OF LIQUID INTERFACES. Journal de Physique Colloques, 1983, 44 (C10), pp.C10-155-C10-161. 10.1051/jphyscol:19831031 . jpa-00223488

\section{HAL Id: jpa-00223488 https://hal.science/jpa-00223488}

Submitted on 1 Jan 1983

HAL is a multi-disciplinary open access archive for the deposit and dissemination of scientific research documents, whether they are published or not. The documents may come from teaching and research institutions in France or abroad, or from public or private research centers.
L'archive ouverte pluridisciplinaire HAL, est destinée au dépôt et à la diffusion de documents scientifiques de niveau recherche, publiés ou non, émanant des établissements d'enseignement et de recherche français ou étrangers, des laboratoires publics ou privés. 


\title{
LIGHT SCATTERING AND REFLECTIVITY OF LIQUID INTERFACES
}

\author{
D. Langevin and J. Meunier \\ Laboratoire de Spectroscopie Hertzienne de Z'T.N.S., 24, we Lhomond, \\ 75231 Paris Cedex 05, France
}

\begin{abstract}
Rêsumé - I'interface entre deux fluides n'est jamais rigoureusement plat, du fait de l'agitation thermique. Ceci modifie les propriétés de réflectivité de l'interface et donne naissance à une diffusion de la lumière.
\end{abstract}

La dépendance en fréquence de la lumière diffusée par les rugosités de surface contient d'intéressantes informations sur la propagation des ondes capillaires. Ceci constitue une méthode très fiable de mesure d'un certain nombre de propriétés de l'interface : tension superficielle, élasticité et viscosité de surface.

La réflectivité de l'interface diffère de la réflectivité de Fresnel à cause
de l'existence, non seulement d'un profil "intrinsèque" de densité au travers
de l'interface, mais également des rugosités de surface. Les différences. ne
sont habituellement importantes qu'au voisinage de l'angle de Brewster. Mais,
lorsque la tension interfaciale est très faible elles peuvent s'observer faci-
lement autour de l'incidence normale. Le problème de la séparation des deux
contributions : épaisseur "intrinsèque" et rugosités, est encore mal résolu.

Une prêsentation de ces divers effets de l'agitation thermique aux interfaces liquides sera donnée.

Abstract - Because of thermal motion, a liquid surface is never perfectly flat. This modifies the relectivity properties of the surface and gives rise to scattering of light.

The frequency dependence of the light scattered by the surface corrugations contains interesting informations about capiliary waves propagation. Several surface properties can be thus measured : surface tension, surface elasticity and viscosity.

The surface reflectivity differs from the Fresnel reflectivity not only because of the "intrinsic" density profile across the intexface, but also because the surface corrugations are present. The differences are usually only important around the Brewster incidence. But when the surface tension is small they can be easily observed around the normal incidence.

A brief summary of these differents aspects of the thermal motion on liquid surfaces will be presented.

\section{I - INTRODUCTION}

The scattering of light from thermally excited surface waves was first predicted by v. Schmoluchowsky ( $\left.{ }^{l}\right)$ in 1908 . He noted that thermal motion should constantly distort the surface and gives rise to a certain roughness. In 1913, L. Mandelstam ( ${ }^{2}$ ) derived the mean square amplitude of the thermal fluctuations and, by using Rayleigh's theory for the diffuse reflection by a rough surface, he calculated the intensity of the scattered light. He qualitatively verified these predictions, from visual observations of the light scattered by a carbon disulfide-methanol interface 
near the critical point. But the conventional spectral lamps made the light scattering experiments difficult to perform. In particular, far from critical points, the surface light scattered intensity can not be distinguished from the light scattered by the bulk of the liquid phases, which is usually much larger. The expeximental papers on the subject were then scarce until the appearance of Helium-neon lasers around 1965. Lasers made possible the frequency analysis of the scattered light. The distinction between bulk and surface scattering became then feasible : indeed the characteristic frequencies and dispersion relations are very different in the two cases.

The first experiments using light beating spectroscopy were performed in our laboratory in $1968\left({ }^{3}\right)$. At the same time katyl and Ingard $\left(^{4}\right)$ obtained independently similar results under more difficult experimental conditions with a Fabry-Perot interferometer. The light beating spectroscopy is much better suited for surface light scattering experiments and was adopted in all further studies.

The technique has now be widely used. A brief summary of both theory and expeximents will be given in the following.

The scattering of light is expected to give rise to a reflectivity loss, that should increase close to a critical point where the scattered intensity becomes very large. This effect was investigated by webb and collaborators since $1965\left(^{5}\right)$. But they attributed the reflectivity loss to the diffuseness of the interface xathex than to its roughness. Indeed the interface between two fluids is never either perfectly flat, nor perfectly sharp. As a critical point is approached, the interface thickens and becomes extremely diffuse, until at the critical point, it disappears completely.

More recently $\left({ }^{6}\right)\left({ }^{7}\right)$ the problem of the reflectivity of a rough and diffuse interface was reconsidered. We have shown in particular that the earlier experiments are in better agreement with the theories when the roughness contribution is properly taken into account. Unfortunately, the complete calculation is only easy for the normal incidence and therefore only applies for very thick interfaces where reflectivity losses are observable.

A brief summary of the existing theories and experiments will also be given hereafter.

II - IIGHT SCATTERING BY LIQUID SURFACES

The vertical displacement of a given point of the surface $\vec{r}$ and at a given instant $t$ can be written as a sum of Fourier components

$$
\zeta(\vec{r}, t)=\sum_{\vec{q}} \underset{q}{q}(t) e^{i \vec{q} \vec{r}}
$$

Each component $\zeta_{\mathrm{q}}$ behaves like a sinusoidal diffraction grating. When $q$ is smallex than $\mathrm{k}$, the wave vector of the incident light, the grating scatters the light. The zeroth order contributes to the regular reflection and the first order to the scattering. The higher orders also give a contribution to the scattering, which is negligible compared to the first order one, because the roughness amplitude is very small (typically $\left\langle\zeta^{2}\right\rangle^{1 / 2} \sim 10 \AA$ ). The contribution of the higher orders to the reflection will be considered later on ( $\S$ III).

The light scattered by a particular Fourier component $\zeta_{\vec{q}}$ is found then in a welldefined direction simply related to $\vec{q}$ by :

$$
\overrightarrow{\mathrm{k}}_{\mathrm{d} \Sigma}-\overrightarrow{\mathrm{k}}_{\Sigma}= \pm \overrightarrow{\mathrm{q}}
$$

Where $\vec{k}_{d \Sigma}$ and $\vec{k}_{\Sigma}$ are, respectively, the projections of the wave vectors of the scattered and reflected light on the surface plane.

The creation of a surface vibration mode of wave vector $\vec{q}$ (surface ripplon) requires 
a certain amount of work against gravity and capillary forces. The energy equipartition theorem leads to

$$
\left\langle\zeta_{q}^{2}\right\rangle=\frac{k_{B} T}{\gamma q^{2}+\left(\rho_{2}-\rho_{1}\right) g}
$$

where $\gamma$ is the surface tension, $\rho_{1}$ and $\rho_{2}$ the densities of the upper and lower fluids respectively. The gravity term $\left(\rho_{1}^{2}-\rho_{2}\right)$ g is usually negligible for the wave vectors of interest in light scattering. It follows that the intensity $I$ of the scattering, which is proportional to $\left\langle\zeta_{\mathrm{q}}^{2}\right\rangle$, becomes very large if the surface tension is small. This happens for instance close to a critical point and give rise to "surface opalescence".

It follows also from equation (2) that I decreases very rapidly when the scattering angle increases. In practice, the experiments are often restricted to very small

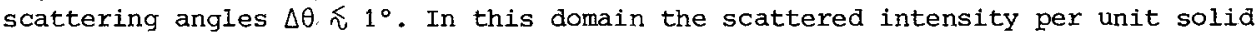
angle around the reflected beam is

$$
\frac{d I}{d \Omega}=I_{0} \frac{k^{4}}{4 \pi^{2}}\left\langle\zeta_{q}^{2}\right\rangle R \cos \theta \quad ; \quad q=k \Delta \theta
$$

where $I_{0}$ is the incident light intensity, $R$ the reflection coefficient, $\theta$ the angle of reflection.

Let us estimate the intensity in the case of the free surface of water $\gamma=72 \mathrm{dyn} / \mathrm{cm}$ $\rho=1 \mathrm{~g} / \mathrm{cm}^{3}$. If we use an He-Ne laser $\left(\mathrm{k} \sim 10^{5} \mathrm{~cm}^{-1}\right)$, an incident beam normal to the surface $\theta=0$, and if we detect the scattered light in the direction $\Delta \theta=10^{\prime}$, then

$$
\frac{1}{I_{0}} \frac{d I}{d 2} \sim 10^{-3}
$$

This value is large compared with the scattering by density fluctuations in the bulk water ( $\sim 10^{-5}$ for a scattering volume $1 \mathrm{~cm}$ thick). But the surface scattering decreases very rapidly as the scattering angle increases, whereas the bulk scattering remains constant.

In order to separate the surface light scattering from the bulk scattering or from the stray light (at low scattering angle) a spectral analysis is required most of the time. We have seen that the angular dependence of the scattered intensity reflects the spatial distribution of the fluctuations $\zeta$. The spectral analysis will reflect their temporal evolution :

$$
I\left(\vec{k}_{d}, \omega_{d}\right)=F \quad F(\vec{q}, \omega) \quad \omega=\omega_{d}-\omega_{0}
$$

$\omega_{0}$ being the frequency of the incident light. $F$ depends only on the incident beam properties and scattering geometry, $P$ only on the scattering system: $P$ is the space time Fourier transform of the correlation function of $\zeta$, i.e., $\left\langle\zeta(\vec{x}, t) \zeta\left(\vec{r}^{*}, t^{\prime}\right)\right\rangle$.

After its creation, a surface ripplon will tend to disappear under the influence of restoring forces (capillarity + gravity) and damping forces due to bulk viscosity in the case of simple fluids. Depending on whether the restoring force is larger or smaller than the damping force, the surface ripplon will propagate as a damped oscillation or will be damped exponentially without propagation. It behaves optically as a moving diffraction grating and the scattered light is frequency shifted by the Doppler effect. If the fluid viscosities $\eta_{1}$ and $\eta_{2}$ are small, the frequency of propagation $\omega_{q}$ and the lifetime of surface "ripplons, $\tau_{q}$ are (propagating case)

$$
\omega_{\mathrm{q}}=\left(\frac{\gamma}{\rho_{1}+\rho_{2}}\right)^{1 / 2} \mathrm{q}^{3 / 2} \quad \frac{1}{\tau_{\mathrm{q}}}=\Delta \omega_{\mathrm{q}}=2 \frac{\left(\eta_{1}+\eta_{2}\right)}{\rho_{1}+\rho_{2}} q^{2}
$$

and if the fluid viscosities are large, or if the surface tension $y$ is very small, their lifetime is (overdamped case) : 


$$
\frac{1}{\tau_{q}}=\Delta \omega_{q}=\frac{\gamma q}{2\left(\eta_{1}+\eta_{2}\right)}
$$

As a consequence, the spectrum as the scattered light in the first case has two Lorentzian components symetric with respect to $\omega_{0}$. They are centered at " $\omega_{0} \pm \omega_{\alpha}$, and their half width is $\Delta \omega_{\mathrm{g}}(\mathrm{eq} 3)$. In the second case, the spectxum is a singlé Lorentzian curve centered at $\omega_{0}$, of half width $\Delta \omega_{q}$ (eq 4 ).

In the intermediate viscosity range, which is encountered vexy often experimentally, the spectrum is much more complex. In a first approximation, the surface can be replaced by a thin elastic membrane having the same vibration modes $\left({ }^{8}\right)$. The spectrum is then identical to that of an harmonic oscillator. Closer to the transition between propagating and overdamped regime, the exact theoretical spectrum $\left({ }^{9}\right)$, still more complex, has to be used. Indeed the coupling between the surface and the bulk fluids becomes then important.

The study of the spectrum of the light scattered by surfaces of simple fluids allows the determination of the surface tension $\gamma$ and of the bulk viscosity $n$. Many others, often more precise methods can be used to measure these quantities. The advantages of the light scattering techniques are:

- the system us at thermal equilibrium,

- the only perturbation to the surface is the light beam whose intensity can be lowered if necessary.

The technique can therefore be used when more simple methods may fail. It can also allow the measurement of different parameters in more complex systems. The following list will review briefly the experiments performed in recent years.

Suxface tension measurements

Critical points pure fluids $\mathrm{CO}_{2}\left({ }^{10}\right)$. Xe $\left({ }^{11}\right) \mathrm{SF}_{6}\left({ }^{12}\right)$

Nematic liquid crystals $\left({ }^{14}\right)$;

$$
\text { binary mixtures cyclohexane-methanol }\left(^{13}\right) \text {; }
$$

Nematic isotropic interface (í)

Lipid bilayers $\left({ }^{16}\right)\left({ }^{17}\right)$;

Multiphase microemulsion systems $\left({ }^{18}\right)$.

\section{other surface forces}

Surface elasticity : insoluble monolayers on water $\left.\left({ }^{19}\right)-{ }^{22}\right)$ interactions between two surfactant layers in soap films (electrostatic, van der waals forces) $\left({ }^{23}\right)\left({ }^{24}\right)$.

BuLk viscosity

Nematic liquid crystals,

Polymer solutions $\left({ }^{25}\right)\left({ }^{26}\right)$.

critical points of pure fluids.

Surface viscosities

Insoluble monolayers on water,

Lipid bilayers.

III - REFLECTIVITY OF LIQUID SURFACES

As pointed out before, the surface corrugations can lower the reflectivity of a liquid. surface.

For the Fourier components of the surface displacement $\zeta_{q}$ of wave vectors such as $\mathrm{q}<\mathrm{k}$ (eq 1), the reflectivity loss is due to the scattering. A very good approximation of the new reflectivity can be obtained when the incidence is normal, by gene- 
ralizing $\left({ }^{6}\right)$ a result of Beckman and Spizzichino $\left({ }^{27}\right.$ ) for perfectly reflecting rough surfaces :

$$
R_{1}=R_{F} e^{-4 k^{2}\left\langle\zeta_{1}^{2}\right\rangle}
$$

where $R_{F}$ is the Fresnel reflectivity and $\left\langle\zeta_{1}^{2}\right\rangle=\int_{0}^{k}\left\langle\zeta_{q}\right\rangle^{2} \frac{\text { gdq }}{2 \pi}$ represents the contribution of the wave vectors $0<q<\mathrm{k}$ in the mean square displacement. The remaining contribution is : $\left\langle\zeta_{2}^{2}\right\rangle=\int_{\mathrm{k}}^{\mathrm{q}_{\max }}\left\langle\zeta_{\mathrm{q}}^{2}\right\rangle \frac{\mathrm{qdq}}{2 \pi}$, where the cut off wave vector $q_{\max }$ is related to the effective interfacial thickness $L: q_{\max } \sim \pi / L$. The contribution $\left\langle\zeta_{2}^{2}\right\rangle$ does not lead to scattering, but to evanescent waves that lower further the reflectivity. The work of $P$. Bousquet $\left({ }^{28}\right)$ relative to diffraction gratings of periodicities smaller than $\lambda=2 \pi / \mathrm{k}$ gives the corresponding reflectivity loss to the first order in $\left\langle\zeta_{2}^{2}\right\rangle$.

A more complete result can be obtained independently with an "effective medium" type theory. Indeed, the sharp but rough profile can be replaced by a space averaged density profile (29)

$$
\rho(z)=\frac{1}{2}\left\{\left(\rho_{1}+\rho_{2}\right)+\frac{1}{\sqrt{\pi}}\left(\rho_{1}-\rho_{2}\right) \int_{0}^{z / L \sqrt{2}} e^{-t^{2}} d t\right\}
$$

where $L$ is the mean square displacement $L=\left\langle\zeta_{2}^{2}\right\rangle^{1 / 2}$.

The reflectivity of the equivalent diffuse interface is given by $\left(^{30}\right)$ :

$$
R=R_{F}\left[\frac{1}{\rho_{1}-\rho_{2}} \int_{\infty}^{\infty} \frac{d \rho}{d z} e^{2 i k z} d z\right]^{2}
$$

one obtains finally:

$$
\mathrm{R}_{2}=\mathrm{R}_{\mathrm{F}} \mathrm{e}^{-4 \mathrm{k}^{2}<\zeta_{2}^{2}>}
$$

Generalizing further the result of Beckman and spizzichino one can replace in equation 5 the reflectivity $R_{F}$ by the reflectivity $R_{2}$ corrected for the contributions of large wave vectors (eq 8). Finally the complete expression of the reflectivity is :

$$
\mathbf{R}=\mathbf{R}_{\mathbf{F}} e^{-4 \mathrm{k}^{2}\left\langle\zeta^{2}\right\rangle} \quad\left\langle\zeta^{2}\right\rangle=\frac{\mathrm{k}_{B} \mathrm{~T}}{4 \pi \gamma} \ln \left[\frac{q_{\max }}{\left(\rho_{1}-\rho_{2}\right) \mathrm{g}}+1\right]
$$

Let us note that Buff, Lovett and stillinger already obtained this result by replacing the whole fluctuations by their space averaged profile = eq 6 with $\mathrm{L}=\left\langle\zeta^{2}\right\rangle{ }^{1 / 2}$. However this procedure is not rigourous since effective medium theories are not appropriate to describe fluctuations of wavelengths larger than $\lambda$ : in particular they do not predict any scattered light.

Let us also note that the derivation of Zielinska, Bedeaux and vlieger $\left({ }^{31}\right)$ for all incidence angles but to the first order in $\left\langle\zeta^{2}\right\rangle$ is also in agreement with eq 9 .

When eq 9 is fitted with the existing experimental data, a good agreement is found for multiphase microemulsion systems $\left({ }^{6}\right)$, but serious discrepancies arise in the case of pure fluids or binary mixtures close to a critical point $\left({ }^{30}\right)\left({ }^{32}\right)$.

It is then tempting to attribute these discrepancies to an "intrinsic density profile" across the interface. The interface will then be both rough and diffuse.

The reflectivity loss associated with a density profile depends on the detailed shape of this profile. The most accurate description of the interface between two fluids close to a critical point is expected to be that proposed by Fisk and widom 
$\left({ }^{33}\right)$ which is a refined form of the van der waals profile $\left({ }^{34}\right)$ :

$$
\rho(z)=\frac{1}{2}\left\{\left(\rho_{1}+\rho_{2}\right)+\left(\rho_{1}-\rho_{2}\right) \frac{\sqrt{2} \text { th } x / L_{p}}{\left[3-\operatorname{th}^{2} x / L_{p}\right] / 2}\right\}
$$

where $L_{p} \sim 2 \xi$, $\xi$ being the correlation lenght for the concentration fluctuations in the coexisting bulk phases.

It has been noted by Webb and collaborators that the Fisk and Widom profile (eq 10) gives reflectivity values close to the error function profile ones (eq 6) :

$$
R \simeq R_{F} e^{-4 k^{2} L_{p}^{2}}
$$

By generalizing again eq 9 one then obtains a very good approximation for the reflectivity of a both rough and diffuse interface ( $\left.{ }^{6}\right)$ :

$$
R=R_{F} e^{-4 k^{2} L^{2}} \quad L^{2}=L_{p}^{2}+\left\langle\zeta^{2}\right\rangle
$$

Coming back to the experimental data, the reflectivity values can now be well accounted for by using eq 11 with $\mathrm{L}_{\mathrm{p}} \sim 2 \xi, \xi$ being deduced from bulk light scattexing measurements. Eq 9 is convenient for microemulsions systems because in these systems : $L_{p} \ll\left\langle\zeta^{2}\right\rangle 1^{2} \succsim L$, whereas for simpler fluids the current situation is $I_{\mathrm{p}} \sim\langle\zeta\rangle^{1 / 2}, i . e$. roughness and diffuseness contribution are equivalent.

The complete calculation of the reflectivity properties is not yet available for a non zero angle of incidence. Separate calculations have been performed for a rough interface to the first order in $k\left\langle\zeta^{2}\right\rangle^{1 / 2}\left({ }^{31}\right)\left({ }^{35}\right)$ and for a diffuse interface close to the Brewster angle $\left(^{36}\right)$. It is not yet clear whether the ratio of the two contributions is the same for different angles of incidence. Preliminary experiments on microemulsions systems seems to indicate that the roughness contribution is very small around the Brewster angle (D. Beaglehole, M.T. Clarkson, A. Upton, to be published). Further theoretical and experimental work are needed to clarify this problem. The case of the Brewster incidence is specially interesting because it allows to study very small interfacial thicknesses $L \gtrsim 5 \AA$ whereas for a normal incidence L $\succsim 200 \AA$.

Finally, let us point out, that the distinction between roughness and diffuseness is somewhat artificial when $q \sim \mathrm{k}_{\max }$. A coherent description of the interfacial thickness has been proposed recently by Jasnow and Rudnick ( ${ }^{37}$ ). Their result for the normal incidence reflectivity is formally identical to eq 11 . It has the advantage of giving a precise value for equivalent cut-off wave vector $q_{\max }$. The knowledge of $q_{\max }$ is not fundamental for the interpretation of these reflectivity data since it goes into a logarithm (eq 9). But it affects more seriously the reflectivities around the Brewster angle $\left({ }^{35}\right)$. A coherent description of the interfacial profile is then also certainly needed to solve the problem of the $q_{\text {max }}$ determination in the general case.

\section{CONCLUSIONS}

The role of thermal fluctuations at the intexface between two fluids has been analyzed. Part of these fluctuations give rise to a scattering of light. The frequency analysis of the scattered light is a promising technique for the study of surface properties : surface tension, surface viscoelasticity. The whole fluctuations also modify the reflectivity properties of the interface. Excepted for the normal incidence, this effect remains very poorly understood. Although also very promising, the technique is not yet able to give valuable informations about interfacial density profiles. Prior to this, a convenient theoretical description has to be given. 


\section{REFERENCES}

(') VON SCHMOLUCHOWSKI M., Ann. Physik 25 (1908) 225.

( $\left.{ }^{2}\right)$ MANDELSTAM L., Ann. Physik 41 (1913) 609.

$\left({ }^{3}\right)$ BOUCHIAT M.A., MEUNIER J. and BROSSEL J., C.R. Acad. Sci. $266 \mathrm{~B}$ (1968) 301, BOUCHIAT M.A. and MEUNIER J., C.R. ACad. Sci. 266B (1968) 301 .

$\left({ }^{4}\right)$ KATYL R.H. and INGARD U., Phys. Rev. Lett. $20 \overline{(1968)} 248$.

$\left({ }^{5}\right)$ GILMER G.M., GILMORE W., HUANG J. and WEBB W.W., Phys. Rev. Lett. 14 (1965) 491

$\left({ }^{6}\right)$ MEUNIER J., C.R. Acad. Sci. 292 II (1981) 1469, MEUNIER J. and LANGEVIN D., J. Phys. Lett. 43 (1982) L-185.

(') BEAGLEHOLE D., Physica 100B (1980) 163.

$\left({ }^{8}\right)$ MEUNIER J., CRUCHON D. and BOUCHIAT M.A., C.R. Acad. Sci. 268B (1969) 422.

$\left({ }^{9}\right)$ BOUCHIAT M.A. and MEUNIER J., J. de Phys. 32 (1971) 561.

$\left({ }^{10}\right)$ MEUNIER J., J. de Phys. 30 (1969) 933, HERPIN J.C. and MEUNIER J., J. de PhYs. 35 (1974) 847.

(11) ZOLLWEG J., HAWKINS G. and BENEDEK G., Phys. Rev. Lett. 27 (1971) 1182.

$\left({ }^{12}\right)$ WU E.S. and WEBB W.W., Phys. Rev. A8 (1973) 2070.

( ${ }^{3}$ ) HUANG J.S. and WEBB W.W., Phys. Rev. Lett. 23 (1969) 160.

$\left(\begin{array}{l}14 \\ (15\end{array}\right)$ LANGEVIN D., J. de Phys. 33 (1972) $249 ; 36$ (1975) 745.

( ${ }^{15}$ ) LANGEVIN D. and BOUCHIAT M.A., Mol. Cryst. Liq. Cryst. 22 (1973) 317.

$\left({ }^{16}\right)$ GRALOWSKI E. and COWEN J.A., Biophysical J. 18 (1977) 23 .

( $\left.{ }^{17}\right)$ GRILLY J.F. and EARNSHAW J.C., Biophysical J. 41 (1983) 197, 211.

$\left({ }^{18}\right)$ POUCHELON A., MEUNIER J., LANGEVIN D. and CAZABAT A.M., J. de Phys. Lett. 41 (1980) L-239, CAZABAT A.M., LANGEVIN D., MEUNIER J. and POUCHELON A., Adv. Coll. Int. Sci. 16 (1982) 175.

(19) HARD 5 . and LOFGREN H., J. Coll. Int. Sci. 60 (1977) 529.

$\left({ }^{20}\right)$ LANGEVIN D., J. Coll. Int. Sci. 80 (1981) $4 \overline{12}$.

( $\left.{ }^{21}\right)$ BYRNE D. and EARNSHAW J.C., J. PhyS. D 12 (1979) 1145.

$\left({ }^{22}\right)$ HARD S. and NEUMAN R.D., J. Coll. Int. SCi. 83 (1981) 315.

$\left({ }^{23}\right)$ VRIJ A., J. Coll. Sci. 19 (1964) 1, FIJNAUT H.M. and JOOSTEN J.G.H., J. Chem. Phys. 69 (1978) 1022, VRIJ A., JOOSTEN J.G.H. and FIJNAUT H.M., Adv. Chem. Phys. 48 (1981) 329.

$\left(\begin{array}{l}24 \\ 2\end{array}\right)$ YOUNG C.Y. and CLARK N.A., J. Chem. Phys. 74 (1981) 4171.

$\left({ }^{25}\right)$ LUNDSTROM I. and MCQUEEN D., J.C.S. Faraday I, 70 (1974) 2351.

$\left({ }^{26}\right)$ IANGEVIN D. and MEUNIER J., in "Photon Correlation Spectroscopy and velocimetry", ed. H.z. Cummins and E.R. Pike, Plenum Press (1977).

$\left({ }^{27}\right)$ BECKMANN P. and SPIZZICHINO A., "The scattering of Electromagnetic waves from Rough Surfaces", Pergamon Press (1963).

$\left({ }^{28}\right)$ BOUSQUET P., Rev. Opt. 41 (1962) 277.

$\left({ }^{29}\right)$ BUFF F.P., LOVETT R.A. and STILLINGER F.H. Jr, Phys. Rev. Lett. 15 (1963) 621.

$\left({ }^{30}\right)$ WU E.S. and WEBB W.W., Phys. Rev. A8 (1973) 2065.

$\left({ }^{31}\right)$ ZIELINSKA B.J.A., BEDEAUX D. and VLIEGER J., Physica 107A (1981) 91 and $117 \mathrm{~A}$ (1983) 28 .

$\left({ }^{32}\right)$ HUANG J.S. and WEBB W.W., J. Chem. Phys. 50 (1969) 3677.

$\left({ }^{33}\right)$ FISK S. and WIDOM B., J. Chem. Phys. $50(\overline{1969)} 3219$.

$\left({ }^{34}\right)$ VAN DER WAALS J., Z. Phys. Chem. 13 (1894) 657.

$\left({ }^{35}\right)$ BEAGLEHOLE D., Physica $112 \mathrm{~B}$ (1982) 320 .

$\left({ }^{36}\right)$ See for instance, BEAGLEHOLE D., these proceedings.

$\left({ }^{37}\right)$ JASNOW D. and RUDNICK J., Phys. Rev. Lett. 41 (1978) 698. 